\title{
Determination of Collision Cross Sections of Protein Ions in an Orbitrap Mass Analyzer
}

\author{
James D. Sanders, ${ }^{1}$ Dmitry Grinfeld, ${ }^{2}$ Konstantin Aizikov, ${ }^{2}$ Alexander Makarov, ${ }^{2}$ Dustin D.
} Holden and Jennifer S. Brodbelt ${ }^{1 *}$

\author{
${ }^{1}$ Department of Chemistry \\ University of Texas at Austin
}

Austin, TX 78712

\author{
${ }^{2}$ Thermo Fisher Scientific \\ 28199 Bremen, Germany
}

1

${ }^{*}$ Correspondence to: Jenny Brodbelt, jbrodbelt@cm.utexas.edu

\section{Abstract:}

We demonstrate a method for determining the collision cross sections (CCS) of protein ions based on the decay rate of the time domain transient signal from an Orbitrap ${ }^{\mathrm{TM}}$ mass analyzer. Multiply charged ions of ubiquitin, cytochrome $\mathrm{c}$ and myoglobin were generated by electrospray ionization of both denaturing solutions and ones with high salt content to preserve native-like structures. A linear relationship between the pressure in the Orbitrap analyzer and the transient decay rate was established and used to demonstrate that the signal decay is primarily due to ionneutral collisions for protein ions across the entire working pressure range of the instrument. The

2 CCSs measured in this study were compared with previously published CCS values measured by 23 ion mobility mass spectrometry (IMS), and results from the two methods were found to differ by 24 less than 7\% for all charge states known to adopt single gas-phase conformations. 


\section{Introduction}

Mass spectrometry provides a wealth of information about the identities, sequences, and modifications of proteins in an efficient high throughput manner. ${ }^{1-3}$ While this information is critical to answering many biologically relevant questions, it is rarely sufficient to reveal the structure/function landscape of proteins and protein complexes. The way that proteins fold into complex tertiary structures and how these structures change in response to environmental conditions and interactions with other molecules is of immense interest to a wide variety of disciplines ranging from fundamental structural biology to drug discovery and development. ${ }^{4}$ Additional insight about the structures of proteins and protein complexes can be obtained by measuring the rotationally averaged collision cross section (CCS), ${ }^{5-8}$ defined as the area of a circle with a radius equal to the sum of the radii of two colliding molecules. If changes in protein conformation manifest as changes in the overall size and shape of the molecule, they can often be detected as changes in CCS.

With mass accuracy and resolving power rivaled only by Fourier Transform ion cyclotron resonance (FT-ICR) instruments, ${ }^{10,11}$ the Orbitrap ${ }^{\mathrm{TM}}$ mass analyzer has rapid emerged as one of the most popular platforms for numerous MS applications ${ }^{9,12-18}$ Sample preparation and ionization techniques that maintain aspects of native protein conformations in the gas phase and newer activation methods such as ultraviolet photodissociation (UVPD) ${ }^{17-21}$ and electron capture dissociation $(\mathrm{ECD})^{22-24}$ now allow the interrogation of protein tertiary structure and conformational changes in addition to sequence information normally associated with MS-based proteomic methods. The coupling of ion mobility (IM) instruments to mass spectrometers has added an additional dimension to the structural characterization of biomolecules by mass spectrometry by facilitating the determination of the CCSs of ions in the gas phase. ${ }^{7,25-28}$ The ability to make such measurements has facilitated numerous studies where CCS measurements of protein ions have been utilized to better understand the dynamics of gas-phase protein ions ${ }^{29-31}$ and to infer conformational changes resulting from chemical modifications, ${ }^{32}$ solution conditions, ${ }^{8}$ as well as protein-protein and protein ligand interactions. ${ }^{7,33}$

While IM-MS remains the most popular strategy for CCS measurements, considerable effort has been dedicated to development of alternative methods that do not require a dedicated mobility cell. The correlation between an ion's CCS and the rate at which the time-domain signal 
1 decays in ICR mass analyzers has long been recognized, ${ }^{34-37}$ and several methods have recently 2 capitalized on this relationship. ${ }^{38}$ For example, CCS measurements of crown ethers and amino acids have been made based on the frequency domain spectral linewidths obtained under elevated

4 pressure conditions; a method dubbed "CRAFTI" (cross-sectional areas by Fourier transform ion 5 cyclotron resonance). ${ }^{39-42}$ A similar technique, performed using a custom-built FT-ICR featuring a more powerful magnet ( $9.4 \mathrm{~T}$ instead of $4.7 \mathrm{~T}$ ), was used to measure CCS of biomolecules up to the size of ubiquitin $(8.5 \mathrm{kDa})$, which to our knowledge is the largest analyte measured by this 8 type of method so far. ${ }^{43}$ This approach directly measured the decay rate of the time-domain transient signal by performing a series of digital low-pass filtering and down-sampling steps followed by fitting an exponential decay function to the resulting decay profile. ${ }^{43,44}$

More recently, other mass spectrometers have been utilized for CCS measurements. For instance, CCSs of several tetraalkylammonium cations and small peptides were measured from spectral linewidths obtained on a home-built FT electrostatic linear ion trap (FT-ELIT) with impressive accuracy. ${ }^{45}$ In another study, a charge-detection mass spectrometer (CDMS) was used to measure CCSs of proteins as large as bovine serum albumin. ${ }^{46}$ The transient signal decay rate in the Orbitrap mass analyzer was shown to be related to ion CCS, although without the ability to directly measure the pressure of background gas in the analyzer chamber, only relative measurements could be made. ${ }^{47}$ Here, we describe a method inspired by this previous study to measure the CCSs of protein ions using an Orbitrap platform. Using this technique, CCSs can be determined directly from the decay rate of an FT transient signal obtained under pressure settings and operating conditions typical of high-resolution top-down proteomics experiments, enabling the nearly seamless addition CCS measurements to existing workflows. Central to this method is a calibration framework designed to determine experimentally background gas pressure in the analyzer chamber which allows the determination of absolute CCS.

\section{Methods}

Equine heart myoglobin, equine heart cytochrome c, and bovine ubiquitin were purchased from Sigma-Aldrich (St. Louis, MO), and LC-MS grade water and methanol were purchased from Merck Millipore (Billerica, MA). Proteins were diluted either in a denaturing 1:1 water-methanol solution containing $0.1 \%$ formic acid to a final concentration of $4 \mu \mathrm{M}$ or in a $200 \mathrm{mM}$ ammonium 
1 The proteins were used as supplied for the denaturing solutions, whereas the proteins were purified

2 using P-6 Bio-Spin columns (Bio-Rad Laboratories, Hercules, CA) for the non-denaturing solutions.

4

\section{Instrumentation}

All experiments were performed on a Thermo Fisher Scientific ${ }^{\mathrm{TM}}$ Orbitrap Elite ${ }^{\mathrm{TM}}$ mass spectrometer (Bremen, Germany) which was modified to incorporate an electronically variable pressure regulator allowing precise control over the pressure of nitrogen collision gas in the HCD ion trapping cell ${ }^{48}$ (this feature is now standard in most Orbitrap mass spectrometers). Since $\mathrm{N}_{2}$ gas leaking from the HCD cell is the primary source of background gas in the Orbitrap analyzer chamber, increasing or decreasing the flow of gas to the HCD cell results in a corresponding increase or decrease of pressure in the Orbitrap chamber, providing a limited means to control the pressure in the Orbitrap analyzer. Ions were generated by direct infusion electrospray ionization either through a heated electrospray ionization source using a spray voltage of $4 \mathrm{kV}$ and a flow rate of $3 \mu \mathrm{L} / \mathrm{min}$ (for denatured samples) or through a gold coated borosilicate emitter using a spray voltage of $0.8-1.2 \mathrm{kV}$. Isolation of individual protein charge states was performed in the dual ion trap using an isolation window of 4-20 m/z. All data was collected at a resolution setting of 480,000@400 m/z,which corresponds to a transient acquisition period of 1.536 seconds.

\section{Transient Data Acquisition and Processing}

A custom license allowing pre-FT transient data to be directly recorded was provided by Thermo Fisher Scientific for the purpose of this study. Data processing was performed using custom scripts written in MATLAB R2016a along with several built-in functions. Briefly, a fast Fourier Transform without apodization or zero filling was used to generate a frequency spectrum from the time domain transient of an isolated charge state. Peaks of interest were shifted to zero frequency, and an inverse Fourier Transform was used to generate a new "filtered" time domain transient signal reflecting only the species of interest. Points were assigned to the maxima of each transient beat, and an exponential decay function was fitted to the points using a least-squares fitting algorithm. The process is summarized in Scheme 1.

To ensure that a consistent number of isotope peaks with high signal to noise were selected across the full range of masses and charge states that were analyzed, a window was defined for 
each charge state that encompassed all FT bins that contained the seven most intense isotope peaks in the isolation spectrum, and these peaks were shifted to near zero frequency. An array of complex zeros was appended to the selected peaks so that the data contained a total of $2^{13} \mathrm{FT}$ bins. While this method relies on access to pre-FT time domain data that requires a special license, it does not require any major instrument modifications aside from the ability to regulate the flow of nitrogen into the HCD cell (a standard feature on most Orbitrap platforms) and can be performed in pressure regimes typical of proteomic and native MS workflows.

Estimation of Gas Pressure in the Orbitrap Analyzer

The background gas in the ultra-high vacuum chamber that houses the Orbitrap mass analyzer is primarily composed of $\mathrm{N}_{2}$ flowing from the HCD cell. While this chamber is equipped with a cold ion gauge to monitor pressure, the pressure inside the Orbitrap analyzer is expected to be somewhat higher owing to the dynamics of collision gas flow from the HCD cell to the Orbitrap chamber as illustrated in Scheme S1 ${ }^{47}$ The pressure gauge readings are also biased by a smaller amount of He originating from the dual linear ion traps and from the atmosphere. Based on such considerations, we expect the pressure of $\mathrm{N}_{2}$ in the ion trap to be $\mathrm{P}[$ orbitrap $]=\mathrm{A} \times \mathrm{P}$ [gauge $]-\mathrm{B}$, where $\mathrm{A}>1, \mathrm{P}$ [gauge] is the gauge reading, and $\mathrm{B}$ accounts for the partial pressure of $\mathrm{He}$ and other possible background gases in the chamber.

To obtain a more accurate estimate of pressure for calculation of CCS, the 9+ charge state of ubiquitin, which is reported to adopt a single gas-phase conformation, ${ }^{28,29}$ was selected as the calibration standard. The decay rate of this ion was measured each day that experiments were performed and each time the pressure settings were changed, and was used to calculate a value for the gas molecule density $n$ that was used for all subsequent CCS calculations. The relationship between the gauge reading and the estimated pressure in the Orbitrap analyzer was consistent dayto-day and across the full range of gas in-flow rates used in the experiments. A plot of this relationship is shown in Figure S1. The calibration coefficients were found to be $A=2.15$ and B $=0.11 \times 10^{-10}$ torr.

\section{Results and Discussion}

Overview of ion motion in an Orbitrap analyzer 
Upon application of a high DC potential, ions are accelerated into an Orbitrap analyzer and trapped in simultaneous orbital rotation around a central electrode and axial oscillation along its length. ${ }^{9}$ The axial oscillations occur at a frequency inversely proportional to the square root of the

$4 \mathrm{~m} / \mathrm{z}$ values of the ions and induce an image current in a pair of detecting electrodes, the magnitude

5 of which is proportional to the number of charges in the coherently orbiting ion packet. Depending on their $\mathrm{m} / \mathrm{z}$, ions travel at speeds ranging from 10,000 to over $50,000 \mathrm{~m} / \mathrm{s}$ inside the Orbitrap analyzer. Consequently, even in the ultra-high vacuum environment of the Orbitrap analyzer where

8 pressure is maintained in the $10^{-10}$ torr range, numerous collisions between ions and background gas molecules occur over the detection period (1.536 seconds at a resolution setting of 480,000), and such collisions are usually of sufficient energy to remove the ion from the coherently orbiting ion packet primarily through fragmentation. ${ }^{47}$ Once ions are removed from the packet, they are no longer detected, so the rate of signal decay is related to the rate of collisions. The rate of collisions, and therefore the signal decay rate, increases with increasing ion CCS area, making it possible to calculate ion CCS from the signal decay rate, ion velocity and background gas molecule density that is related to the gas pressure.

\section{Extraction of CCS from transient signal decay rate}

Ions in the Orbitrap mass analyzer possess high kinetic energies (1.5 - $3.6 \mathrm{keV} /$ charge) geometry and independent of its velocity as described by the energetic hard-sphere collision model proposed by Guo et al. ${ }^{44}$ It is further assumed that a single collision is sufficient to remove an ion

22 from the coherently orbiting ion packet either through fragmentation or scattering. This is opposite to the Langevin model in which CCS decreases with increasing velocity due to dipole-induced dipole interactions. Based on these assumptions, the rate at which ions are removed from the population will be equal to the rate of collisions between ions and neutrals. In the D20 model Orbitrap analyzer used in the Orbitrap Elite platform, the ions of mass $m$ and charge $z$ oscillate in the axial direction with the frequency 


$$
f_{z}[H z]=12170(m / Z[D a])^{-1 / 2}
$$

2 and simultaneously revolve around the central electrode. The average ion path per one axial 3 oscillation, that also incorporates the rotation, may be estimated as $L=65.1 \mathrm{~mm}$. Accordingly, the 4 average number (probability) of a collision on a single oscillation is $n L \sigma$ where $n$ is the neutral gas 5 molecule density (molecules $/ \mathrm{m}^{3}$ ) and $\sigma$ is the CCS of the colliding ion-neutral pair. Given the 6 number $N$ of oscillating ions, the number of ions lost during one oscillation period $\Delta t=\frac{1}{f_{z}}$ is $\Delta N=$

$7-n L \sigma N$. Integration eventually gives the exponential time dependence of the number of ions and, 8 therefore, the signal

$$
N(t)=N_{0} e^{-c t}
$$

where $N_{0}=N(0)$ is the number of injected ions and $c$ is the decay constant. Expression for the latter reads

$$
c=n L \sigma f_{z}=\frac{P L f_{z}}{k T} \sigma
$$

where we used the ideal gas thermodynamic equation $P=n k T$ connecting the molecule density $n$ with the pressure $P$ and temperature $T, k$ being the Boltzmann's constant. The reciprocal decay rate is the time constant $\tau=\frac{1}{c}$ over which the signal intensity drops by a factor of $e{ }^{47}$ This value can be determined experimentally in order to evaluate the CCS $\sigma$.

$$
\sigma=\frac{1}{n L f_{z} \tau}
$$

\section{Effect of background gas pressure on transient decay rate}

For ion CCS to be accurately determined by this method, it must be established that the decay of the transient signal is primarily caused by collisions of the protein ions with neutral gas molecules (i.e. collisional damping caused by fragmentation or scattering of ions after collisions with neutrals). To assess the influence of collisional damping and ensure that that the decay of the transient signal is primarily caused by collisions of the protein ions with neutral gas molecules, the pressure in the Orbitrap analyzer was varied by adjusting the pressure in the HCD ion trap and 
monitoring the pressure change in the Orbitrap chamber using the cold ion gauge. Pressure was

2 varied over the range of $0.3 \times 10^{-10}$ to $0.6 \times 10^{-10}$ torr in the Orbitrap chamber (as measured by the cold ion gauge), which roughly corresponds to a calculated pressure range of $0.5 \times 10^{-10}$ to $1.2 \times$ $10^{-10}$ torr in the Orbitrap analyzer (see the experimental section, Figure S1 and Scheme S1 for

5 pressure calibration details). If collisions with neutrals are the primary source of signal decay, a linear relationship between pressure and decay rate that trends toward zero decay at zero pressure would be expected. Any deviation from the linear trend at lower pressures would suggest that other factors such as space charge or field imperfections contribute to non-collisional loss of ions or ion coherence. Figure 1 shows the relationship between gas pressure and the experimentallydetermined decay constant for several charge states of ubiquitin. The linear nature and near-zero intercepts of the trendlines with respect to the calibrated pressure in the Orbitrap analyzer provide compelling evidence that the observed transient decay is primarily due to ion-neutral collisions and thus can be used to determine ion CCS. For the size and mass range of the ions considered in this study, the best results were obtained at a cold ion gauge reading of $0.4 \times 10^{-10}$ torr (corresponding to a calculated pressure of about $0.8 \times 10^{-10}$ torr in the analyzer), and thus all data presented below were collected at that pressure. Figure 2 shows processed transient signals and fitted decay profiles from ubiquitin $(11+)$ collected at four pressure settings that span the practical pressure range of the instrument for a protein of this size. likely need to be increased so that sufficient decay could be observed over the course of the detection period. Achieving sufficiently high pressures in the Orbitrap analyzer by modulating gas

CCS of protein ions

Coulombic repulsion between charge sites on multiply charged protein ions causes unfolding of these ions in the gas phase. This sequential unfolding with increasing charge state, 
documented by numerous IMS studies ${ }^{26,27,29,31,49}$ and provides a convenient means to calibrate and compare various methods for measuring CCS. The CCS values obtained for the most abundant charge states of the three proteins used in this study (ubiquitin, cytochrome c, and myoglobin) are summarized as a function of charge state in Figure 3 and Table S1. As expected, CCS consistently increases with increasing charge state for all three proteins. Interestingly, while the relationship between charge state and CCS is nearly linear in the higher charge state range, the slope of each data series appears to be steeper at lower charge states, particularly for those generated from nativelike solution conditions. While the effect is subtle, it is consistent with other reports that suggest that many elements of protein tertiary structure are preserved in the gas phase for very low charge states but rapidly unfold as charge state increases. ${ }^{50}$ For higher charge states, tertiary structure is largely absent and secondary helical structures begin to unfold as more charges are added, ${ }^{51}$ resulting in the more linear relationship between CCS and charge state observed in this study and others. $^{26,27,29,49}$

\section{Comparison to IMS data}

While CCS measurements in both IMS systems and the Orbitrap analyzer are based on the frequency of ion-neutral collisions, the nature of such collisions differs in several ways between the two platforms. IMS experiments rely on momentum transfer between ions and neutrals during numerous low-energy collisions. Because of the low relative velocities of the ion-neutral pairs, long range interactions can result in momentum transfer between molecules that would not interact under more energetic conditions, resulting in CCS values that are expected to be slightly larger than those calculated by a projection approximation and are more sensitive to other properties such as the temperature and polarizability of the molecules. ${ }^{52}$ This behavior is best described by the Langevin collision model. ${ }^{44}$ In contrast, ion-neutral interactions in the Orbitrap analyzer are characterized by much higher kinetic energies that largely negate the effect of any long-range interactions. This results in collisions best described by the energetic hard-sphere collision model, ${ }^{44}$ in which ion CCS is treated as a constant that is not affected by properties of the collision gas other than its mass. Because of these differences, we expect CCS values measured in the Orbitrap to be systematically smaller than those measured by IMS. However, since the two techniques probe very similar, though not identical, properties of a molecule and share many potential applications, it is useful to compare their results. 
An array of prior IMS studies have shown that while protein ions in the highest and the lowest charge states adopt a single gas-phase conformation, ions in intermediate charge states are found in an distribution of co-existing conformations with different cross-sectional areas. ${ }^{26,27,29,53,54}$ While it is possible to separate ions with the same $m / z$ but different cross-sections

5 by IMS, FT-measurements only provide a single CCS value for all ions of the same $\mathrm{m} / z$ value. This makes direct comparison of Orbitrap and IMS CCS values for charge states known to adopt multiple gas-phase conformations inherently imprecise. Owing to this challenge, ions in higher charge states produced from denaturing solutions and ions in lower charge states from nondenaturing solutions were selected for comparison to IMS data. While all of the high charge states selected for comparison are known to only adopt single or few closely related gas-phase conformations, the low charge states examined for ubiquitin and cytochrome $\mathrm{c}$ (non-denaturing solutions) are known to exist in multiple gas-phase conformations. ${ }^{26,27,29}$ Interestingly, the lowest charge states ubiquitin (5+) and cytochrome c (6+) show significantly less variation in CCS (based on IMS data from ${ }^{26,27,29,53,54}$ ) compared to the adjacent higher charge states. ${ }^{26,27,29}$ For this reason, only the lowest observed charge state of each these two proteins was selected for comparison to IMS data extracted from two studies, ${ }^{55}$ and, ${ }^{25}$ for which a single CCS value calculated from the median IMS drift time was reported for each protein. Finally, since the collision gas in the Orbitrap analyzer is primarily $\mathrm{N}_{2}$, comparative IMS-CCS values obtained using nitrogen as opposed to helium as the drift gas ${ }^{25,49,55}$ were used when possible. When only CCS values measured in He were available, they were adjusted as described in Figure S2 to account for the consistently larger obtained from measuring CCS in $\mathrm{N}_{2}$ as opposed to He.

Figure 4 and Table 1 provide a comparison of CCSs measured using the FT transient decay method to CCSs measured previously by IMS. The CCSs measured for the high charge states of ubiquitin $(8.5 \mathrm{kDa})$ and cytochrome c $(12.4 \mathrm{kDa})$ show good agreement with the CCS values measured by IMS, with relative differences equal to or lower than $5 \%$, which is somewhat greater than the uncertainty of 2-3\% commonly reported for IMS measurements. CCSs measured for myoglobin (17.5 kDa in its heme-bound holo form and $16.9 \mathrm{kDa}$ in the apo form) deviate more significantly from IMS values, with relative differences ranging from 2-7\%. The CCS values measured for myoglobin are also consistently lower than those based on IMS. This outcome could indicate that the assumption that a single collision is always sufficient to remove an ion from the coherently orbiting packet may not be uniformly valid for larger proteins that possess higher 
1 kinetic energies and have more pathways to disperse internal energy acquired through a collision.

2 The upper mass limit of this method and the effects of using heavier or lighter collision gasses, which will change the center of mass collision energy and potentially affect this limit, will be investigated in a future study. conditions (ubiquitin 5+, cytochrome c 6+, and holomyoglobin 8+) show similar deviations from published IMS results. ${ }^{25,27,49,55}$ Higher charge states of ubiquitin $(6+)$ and cytochrome c (7+), both of which are known to adopt numerous gas phase structures that span a large range of CCS values, ${ }^{26,27,29}$ showed considerably more variation in our results, although it should be noted that these ions fall within the ranges published previously. ${ }^{26,27,29}$ While the inability to resolve multiple gas-phase conformations is a limitation of this method, conformational changes in solution induced by events such as the binding of a cofactor or other ligand are often observed as a shift in the global average CCS even when multiple gas-phase structures are present. ${ }^{33,49}$ If the change is sufficiently large it should be detectable by this method, and the application of this method to biologically relevant systems is the subject of ongoing work in our group. It should also be noted that if an ion is removed from the ion packet and no longer detected after the first collision, there is no opportunity for collisional heating to occur in the Orbitrap (though heating can still occur in other portions of the instrument if "harsh" ion optics settings are used). While this hypothesis will require further investigation, it represents a potentially interesting aspect of this method that could prove useful for measuring CCS of less robust analytes. Although this method still provides only relative CCS measurements, this work establishes a framework for these measurements to be related to an appropriate calibration standard and thus estimate an absolute CCS value. These data show that the results are comparable in many ways to IMS measurements provided a suitable calibration 25 standard with known CCS is used.

\section{Conclusion:}

The decay rate of the time domain signal in an Orbitrap mass analyzer can be used to determine the CCS of protein ions generated from both denaturing and native-like solutions. Although this method is not capable of separating multiple co-existing structural conformations 30 (which is an important performance attribute of IMS), it does not require any major instrument 
1 modifications, it can be used prior to or after MS/MS experiments, and it simultaneously allows

2 the acquisition of high resolution mass spectra. While it is not a direct replacement for IMS, this

3 technique represents a significant addition to the FT-MS toolbox by enabling the addition of CCS

4 measurements to existing workflows without modifications to hardware or experimental

5 conditions. Effort to extend the method to other protein-ligand complexes and larger multimeric

6 protein complexes is underway and represents an intriguing new opportunity for expanding the

7 capabilities of Orbitrap mass spectrometers in the context of native MS.

9 ACKNOWLEDGMENTS

10 We acknowledge the following funding sources: NSF (Grant CHE1402753) and the Welch

11 Foundation (Grant F-1155). This material is based upon work supported by the National Science

12 Foundation Graduate Research Fellowship under Grant No. (Grant DGE-1610403) awarded to JS.

13 Development of signal processing algorithms received funding from the European Union's

14 Horizon 2020 research and innovation programme under grant agreement No 686547 (MSMed

15 project).

17 The authors declare following competing financial interests: Some authors are employees of

18 Thermo Fisher Scientific, which manufactures and sells Orbitrap-based mass spectrometers.

20 Supporting Information: The following items are included: a tabulation of CCS values 21 measured in the present study plus reference values from IMS studies; a scheme of the gas flow

22 dynamics of the Orbitrap mass spectrometer; a graph showing the relationship between gauge

23 pressure reading and calculated pressure; and a graphical comparison of CCS values measured in

24 He over $\mathrm{N}_{2}$. 


\begin{tabular}{|c|c|c|}
\hline 1 & \multicolumn{2}{|c|}{ References } \\
\hline & & \\
\hline 3 & (1) & Bensimon, A.; Heck, A. J. R.; Aebersold, R. Mass Spectrometry-Based Proteomics and Network \\
\hline 4 & & Biology. Annu. Rev. Biochem. 2012, 81 (1), 379-405. \\
\hline 5 & $(2)$ & Cox, J.; Mann, M. Quantitative, High-Resolution Proteomics for Data-Driven Systems Biology. \\
\hline 6 & & Annu. Rev. Biochem. 2011, 80 (1), 273-299. \\
\hline 7 & (3) & Mann, M.; Kulak, N. A.; Nagaraj, N.; Cox, J. The Coming Age of Complete, Accurate, and \\
\hline 8 & & Ubiquitous Proteomes. Mol. Cell 2013, 49 (4), 583-590. \\
\hline 9 & (4) & Robinson, C. V.; Sali, A.; Baumeister, W. The Molecular Sociology of the Cell. Nature 2007, 450 \\
\hline 10 & & (7172), 973-982. \\
\hline 11 & (5) & Clemmer, D. E.; Jarrold, M. F. Ion Mobility Measurements and Their Applications to Clusters and \\
\hline 12 & & Biomolecules. J. Mass Spectrom. 1997, 32 (6), 577-592. \\
\hline 13 & (6) & Wyttenbach, T.; von Helden, G.; Bowers, M. T. Gas-Phase Conformation of Biological Molecules: \\
\hline 14 & & Bradykinin. J. Am. Chem. Soc. 1996, 118 (35), 8355-8364. \\
\hline 15 & (7) & Ruotolo, B. T.; Benesch, J. L. P.; Sandercock, A. M.; Hyung, S.-J.; Robinson, C. V. Ion Mobility-mass \\
\hline 16 & & Spectrometry Analysis of Large Protein Complexes. Nat. Protoc. 2008, 3 (7), 1139-1152. \\
\hline 17 & (8) & Vahidi, S.; Stocks, B. B.; Konermann, L. Partially Disordered Proteins Studied by Ion Mobility-Mass \\
\hline 18 & & Spectrometry: Implications for the Preservation of Solution Phase Structure in the Gas Phase. \\
\hline 19 & & Anal. Chem. 2013, 85 (21), 10471-10478. \\
\hline 20 & (9) & Scigelova, M.; Makarov, A. Orbitrap Mass Ana \\
\hline 21 & & PROTEOMICS 2006, 6 (S2), 16-21. \\
\hline 22 & (10) & Nikolaev, E. N.; Kostyukevich, Y. I.; Vladimirov, G. N. Fourier Transform Ion Cyclotron Resonance \\
\hline 23 & & (FT ICR) Mass Spectrometry: Theory and Simulations: FT ICR MS. Mass Spectrom. Rev. 2016, 35 \\
\hline 24 & & $(2), 219-258$. \\
\hline 25 & (11) & Marshall, A. G.; Hendrickson, C. L.; Jackson, G. S. Fourier Transform lon Cyclotron Resonance \\
\hline 26 & & Mass Spectrometry: A Primer. Mass Spectrom. Rev. 1998, 17 (1), 1-35. \\
\hline 27 & $(12)$ & Nagaraj, N.; Alexander Kulak, N.; Cox, J.; Neuhauser, N.; Mayr, K.; Hoerning, O.; Vorm, O.; Mann, \\
\hline 28 & & M. System-Wide Perturbation Analysis with Nearly Complete Coverage of the Yeast Proteome by \\
\hline 29 & & Single-Shot Ultra HPLC Runs on a Bench Top Orbitrap. Mol. Cell. Proteomics 2012, 11 (3), \\
\hline 30 & & M111.013722-M111.013722. \\
\hline 31 & (13) & Geiger, T.; Cox, J.; Mann, M. Proteomics on an Orbitrap Benchtop Mass Spectrometer Using All- \\
\hline 32 & & Ion Fragmentation. Mol. Cell. Proteomics 2010, 9 (10), 2252-2261. \\
\hline 33 & (14) & Jaffe, J. D.; Keshishian, H.; Chang, B.; Addona, T. A.; Gillette, M. A.; Carr, S. A. Accurate Inclusion \\
\hline 34 & & Mass Screening: A Bridge from Unbiased Discovery to Targeted Assay Development for Biomarker \\
\hline 35 & & Verification. Mol. Cell. Proteomics 2008, 7 (10), 1952-1962. \\
\hline 36 & (15) & Rosati, S.; Rose, R. J.; Thompson, N. J.; van Duijn, E.; Damoc, E.; Denisov, E.; Makarov, A.; Heck, A. \\
\hline 37 & & J. R. Exploring an Orbitrap Analyzer for the Characterization of Intact Antibodies by Native Mass \\
\hline 38 & & Spectrometry. Angew. Chem. Int. Ed. 2012, 51 (52), 12992-12996. \\
\hline 39 & (16) & Rose, R. J.; Damoc, E.; Denisov, E.; Makarov, A.; Heck, A. J. R. High-Sensitivity Orbitrap Mass \\
\hline 40 & & Analysis of Intact Macromolecular Assemblies. Nat. Methods 2012, 9 (11), 1084-1086. \\
\hline 41 & (17) & Cammarata, M. B.; Thyer, R.; Rosenberg, J.; Ellington, A.; Brodbelt, J. S. Structural \\
\hline 42 & & Characterization of Dihydrofolate Reductase Complexes by Top-Down Ultraviolet \\
\hline 43 & & Photodissociation Mass Spectrometry. J. Am. Chem. Soc. 2015, 137 (28), 9128-9135. \\
\hline 44 & (18) & Morrison, L. J.; Brodbelt, J. S. 193 Nm Ultraviolet Photodissociation Mass Spectrometry of \\
\hline 45 & & Tetrameric Protein Complexes Provides Insight into Quaternary and Secondary Protein Topology. \\
\hline 46 & & J. Am. Chem. Soc. 2016, 138 (34), 10849-10859. \\
\hline 47 & (19) & Brodbelt, J. S. Photodissociation Mass Spectrometry: New Tools for Characterization of Biological \\
\hline 48 & & Molecules. Chem. Soc. Rev. 2014, 43 (8), 2757. \\
\hline
\end{tabular}


(20) Shaw, J. B.; Li, W.; Holden, D. D.; Zhang, Y.; Griep-Raming, J.; Fellers, R. T.; Early, B. P.; Thomas, P. M.; Kelleher, N. L.; Brodbelt, J. S. Complete Protein Characterization Using Top-Down Mass Spectrometry and Ultraviolet Photodissociation. J. Am. Chem. Soc. 2013, 135 (34), 12646-12651.

(21) Cannon, J. R.; Martinez-Fonts, K.; Robotham, S. A.; Matouschek, A.; Brodbelt, J. S. Top-Down 193Nm Ultraviolet Photodissociation Mass Spectrometry for Simultaneous Determination of Polyubiquitin Chain Length and Topology. Anal. Chem. 2015, 87 (3), 1812-1820.

(22) Zhang, H.; Cui, W.; Gross, M. L. Native Electrospray lonization and Electron-Capture Dissociation for Comparison of Protein Structure in Solution and the Gas Phase. Int. J. Mass Spectrom. 2013, 354-355, 288-291.

(23) Schennach, M.; Breuker, K. Probing Protein Structure and Folding in the Gas Phase by Electron Capture Dissociation. J. Am. Soc. Mass Spectrom. 2015, 26 (7), 1059-1067.

(24) Yin, S.; Loo, J. A. Top-down Mass Spectrometry of Supercharged Native Protein-ligand Complexes. Int. J. Mass Spectrom. 2011, 300 (2-3), 118-122.

(25) Bush, M. F.; Hall, Z.; Giles, K.; Hoyes, J.; Robinson, C. V.; Ruotolo, B. T. Collision Cross Sections of Proteins and Their Complexes: A Calibration Framework and Database for Gas-Phase Structural Biology. Anal. Chem. 2010, 82 (22), 9557-9565.

(26) Shelimov, K. B.; Clemmer, D. E.; Hudgins, R. R.; Jarrold, M. F. Protein Structure in Vacuo : GasPhase Conformations of BPTI and Cytochrome C. J. Am. Chem. Soc. 1997, 119 (9), 2240-2248.

(27) May, J. C.; Jurneczko, E.; Stow, S. M.; Kratochvil, I.; Kalkhof, S.; McLean, J. A. Conformational Landscapes of Ubiquitin, Cytochrome c, and Myoglobin: Uniform Field lon Mobility Measurements in Helium and Nitrogen Drift Gas. Int. J. Mass Spectrom. 2017.

(28) May, J. C.; Morris, C. B.; McLean, J. A. Ion Mobility Collision Cross Section Compendium. Anal. Chem. 2017, 89 (2), 1032-1044.

(29) Valentine, S. J.; Counterman, A. E.; Clemmer, D. E. Conformer-Dependent Proton-Transfer Reactions of Ubiquitin Ions. J. Am. Soc. Mass Spectrom. 1997, 8 (9), 954-961.

(30) Hopper, J. T. S.; Oldham, N. J. Collision Induced Unfolding of Protein Ions in the Gas Phase Studied by Ion Mobility-Mass Spectrometry: The Effect of Ligand Binding on Conformational Stability. J. Am. Soc. Mass Spectrom. 2009, 20 (10), 1851-1858.

(31) Badman, E. R.; Myung, S.; Clemmer, D. E. Evidence for Unfolding and Refolding of Gas-Phase Cytochrome c lons in a Paul Trap. J. Am. Soc. Mass Spectrom. 2005, 16 (9), 1493-1497.

(32) Valentine, S. J.; Anderson, J. G.; Ellington, A. D.; Clemmer, D. E. Disulfide-Intact and-Reduced Lysozyme in the Gas Phase: Conformations and Pathways of Folding and Unfolding. J. Phys. Chem. B 1997, 101 (19), 3891-3900.

(33) Calabrese, A. N.; Speechley, L. A.; Pukala, T. L. Characterisation of Calmodulin Structural Transitions by Ion Mobility Mass Spectrometry. Aust. J. Chem. 2012, 65 (5), 504.

(34) Wobschall, D.; Graham, J. R.; Malone, D. P. Ion Cyclotron Resonance and the Determination of Collision Cross Sections. Phys. Rev. 1963, 131 (4), 1565-1571.

(35) Wobschall, D.; Fluegge, R. A.; Graham, J. R. Collision Cross Sections of Hydrogen and Other lons as Determined by lon Cyclotron Resonance. J. Chem. Phys. 1967, 47 (10), 4091-4094.

(36) Huntress, W. T. Ion Cyclotron Resonance Power Absorption: Collision Frequencies for $\mathrm{CO}_{2}{ }^{+}, \mathrm{N}_{2}{ }^{+}$ , and $\mathrm{H}_{3}{ }^{+}$Ions in Their Parent Gases. J. Chem. Phys. 1971, 55 (5), 2146-2155.

(37) Ridge, D. P.; Beauchamp, J. L. The Interaction of Ions with Nonpolar Neutrals: The Collision Broadening of Ion Cyclotron Resonance Lines of lons in Hydrogen and Methane. J. Chem. Phys. 1976, 64 (7), 2735-2746.

(38) Li, D.; Tang, Y.; Xu, W. Ion Collision Cross Section Measurements in Fourier Transform-Based Mass Analyzers. The Analyst 2016, 141 (12), 3554-3561. 
(39) Yang, F.; Voelkel, J. E.; Dearden, D. V. Collision Cross Sectional Areas from Analysis of Fourier Transform Ion Cyclotron Resonance Line Width: A New Method for Characterizing Molecular Structure. Anal. Chem. 2012, 84 (11), 4851-4857.

(40) Yang, F.; Jones, C. A.; Dearden, D. V. Effects of Kinetic Energy and Collision Gas on Measurement of Cross Sections by Fourier Transform Ion Cyclotron Resonance Mass Spectrometry. Int. J. Mass Spectrom. 2015, 378, 143-150.

(41) Anupriya; Jones, C. A.; Dearden, D. V. Collision Cross Sections for 20 Protonated Amino Acids: Fourier Transform Ion Cyclotron Resonance and Ion Mobility Results. J. Am. Soc. Mass Spectrom. 2016.

(42) Anupriya; Gustafson, E.; Mortensen, D. N.; Dearden, D. V. Quantitative Collision Cross-Sections from FTICR Linewidth Measurements: Improvements in Theory and Experiment. J. Am. Soc. Mass Spectrom. 2017.

(43) Mao, L.; Chen, Y.; Xin, Y.; Chen, Y.; Zheng, L.; Kaiser, N. K.; Marshall, A. G.; Xu, W. Collision Cross Section Measurements for Biomolecules within a High-Resolution Fourier Transform Ion Cyclotron Resonance Cell. Anal. Chem. 2015, 87 (8), 4072-4075.

(44) Guo, D.; Xin, Y.; Li, D.; Xu, W. Collision Cross Section Measurements for Biomolecules within a High-Resolution FT-ICR Cell: Theory. Phys Chem Chem Phys 2015, 17 (14), 9060-9067.

(45) Dziekonski, E. T.; Johnson, J. T.; Lee, K. W.; McLuckey, S. A. Determination of Collision Cross Sections Using a Fourier Transform Electrostatic Linear Ion Trap Mass Spectrometer. J. Am. Soc. Mass Spectrom. 2017.

(46) Elliott, A. G.; Harper, C. C.; Lin, H.-W.; Susa, A. C.; Xia, Z.; Williams, E. R. Simultaneous Measurements of Mass and Collisional Cross-Section of Single lons with Charge Detection Mass Spectrometry. Anal. Chem. 2017, 89 (14), 7701-7708.

(47) Makarov, A.; Denisov, E. Dynamics of lons of Intact Proteins in the Orbitrap Mass Analyzer. J. Am. Soc. Mass Spectrom. 2009, 20 (8), 1486-1495.

(48) Shaw, J. B.; Brodbelt, J. S. Extending the Isotopically Resolved Mass Range of Orbitrap Mass Spectrometers. Anal. Chem. 2013, 85 (17), 8313-8318.

(49) Schenk, E. R.; Almeida, R.; Miksovska, J.; Ridgeway, M. E.; Park, M. A.; Fernandez-Lima, F. Kinetic Intermediates of Holo- and Apo-Myoglobin Studied Using HDX-TIMS-MS and Molecular Dynamic Simulations. J. Am. Soc. Mass Spectrom. 2015, 26 (4), 555-563.

(50) Seo, J.; Hoffmann, W.; Warnke, S.; Bowers, M. T.; Pagel, K.; von Helden, G. Retention of Native Protein Structures in the Absence of Solvent: A Coupled Ion Mobility and Spectroscopic Study. Angew. Chem. Int. Ed. 2016, 55 (45), 14173-14176.

(51) González Flórez, A. I.; Mucha, E.; Ahn, D.-S.; Gewinner, S.; Schöllkopf, W.; Pagel, K.; von Helden, G. Charge-Induced Unzipping of Isolated Proteins to a Defined Secondary Structure. Angew. Chem. Int. Ed. 2016, 55 (10), 3295-3299.

(52) Gabelica, V.; Marklund, E. Fundamentals of Ion Mobility Spectrometry. Curr. Opin. Chem. Biol. 2018, 42, 51-59.

(53) Laszlo, K. J.; Munger, E. B.; Bush, M. F. Folding of Protein lons in the Gas Phase after Cation-toAnion Proton-Transfer Reactions. J. Am. Chem. Soc. 2016, 138 (30), 9581-9588.

(54) Laszlo, K. J.; Buckner, J. H.; Munger, E. B.; Bush, M. F. Native-Like and Denatured Cytochrome c Ions Yield Cation-to-Anion Proton Transfer Reaction Products with Similar Collision CrossSections. J. Am. Soc. Mass Spectrom. 2017, 28 (7), 1382-1391.

(55) Salbo, R.; Bush, M. F.; Naver, H.; Campuzano, I.; Robinson, C. V.; Pettersson, I.; Jørgensen, T. J. D.; Haselmann, K. F. Traveling-Wave Ion Mobility Mass Spectrometry of Protein Complexes: Accurate Calibrated Collision Cross-Sections of Human Insulin Oligomers: Traveling-Wave IM-MS of Protein Complexes. Rapid Commun. Mass Spectrom. 2012, 26 (10), 1181-1193. 
1 (56) Allen, S. J.; Eaton, R. M.; Bush, M. F. Analysis of Native-Like lons Using Structures for Lossless Ion $2 \quad$ Manipulations. Anal. Chem. 2016, 88 (18), 9118-9126.

3 


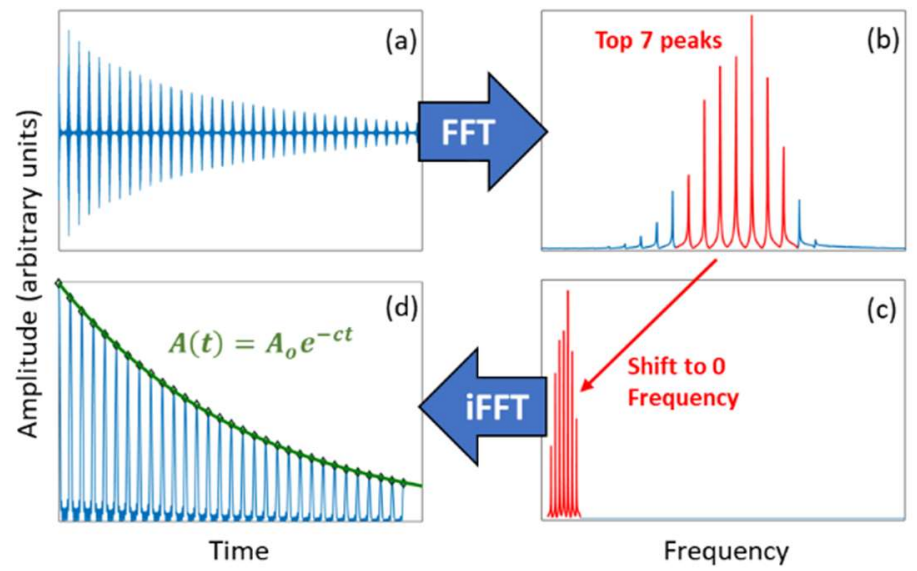

2 Scheme 1: Digital signal processing of the Orbitrap transient signal: (a) The time domain

3 transient signal before processing. (b) The frequency domain spectrum after Fourier

4 transformation of (a), showing the species of interest with the FT bins selected for processing

5 shown in red. (c) The selected FT bins are shifted to 0 frequency and padded with complex zeros

6 to create a total of $2^{13}$ FT bins. (d) Inverse FFT of (c) with the fitted decay profile represented by 7 a green line. 


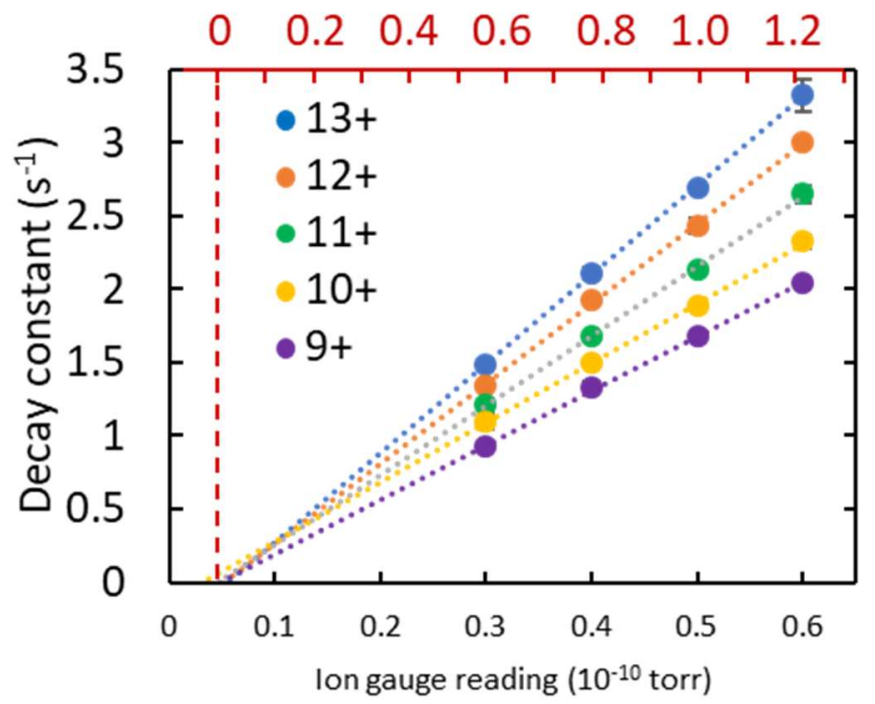

4 Figure 1: Measured decay constants for ubiquitin (9+ through 13+) as a function of background 5 gas pressure as measured by the ion gauge (bottom axis) and in the Orbitrap analyzer (top axis).

6 The coefficient of determination $\left(\mathrm{R}^{2}\right)$ for each fitted trendline is $>0.99$, and the $\mathrm{y}$-intercepts are $<$ $7 \pm 0.1$ with respect to the Orbitrap pressure, indicating that the observed transient signal decay is

8 caused almost exclusively by ion-neutral collisions in the Orbitrap analyzer. The Orbitrap

9 pressure is related to the gauge reading by $P_{\text {Orbitrap }}=2.16 P_{\text {gauge }}+8.74 \times 10^{-12}$ torr 


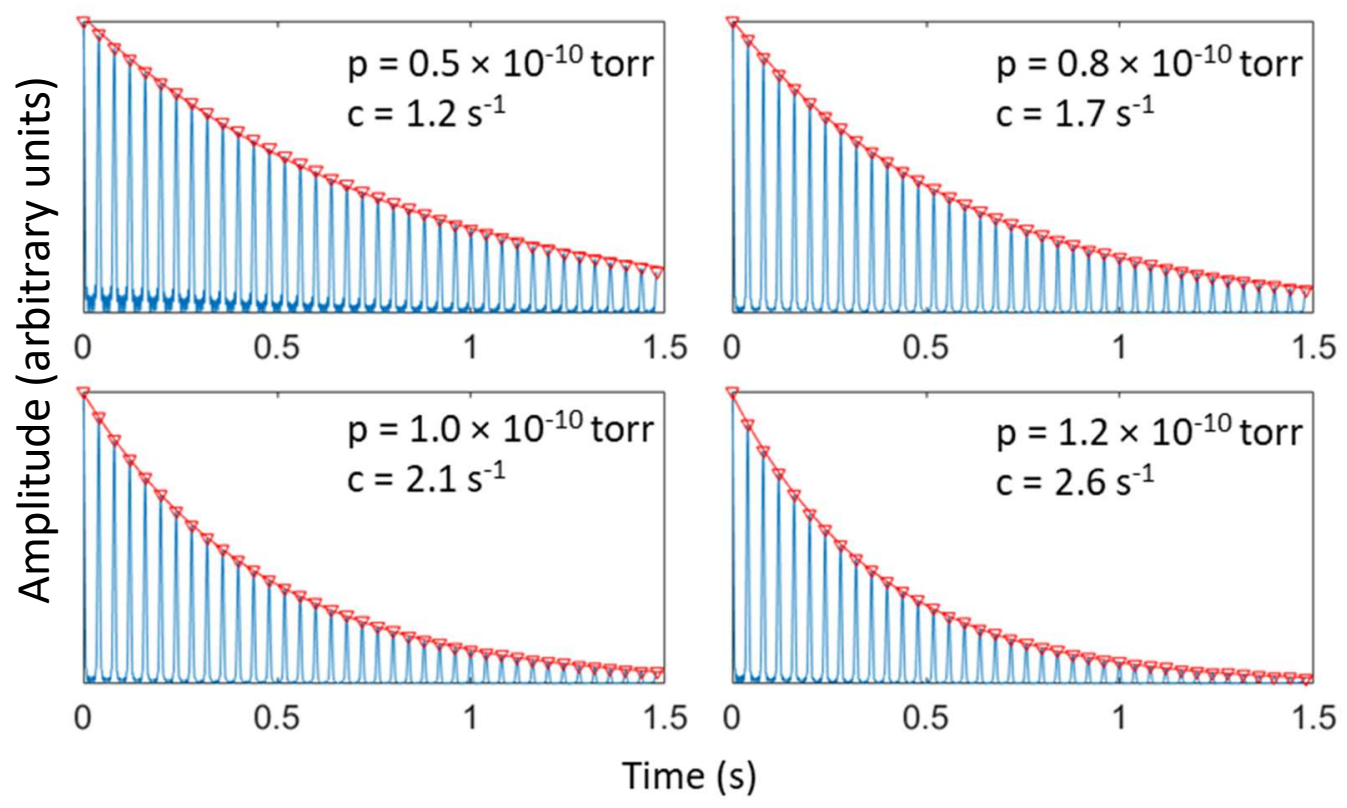

1

2

$3 \quad$ Figure 2: Processed transient signals (blue) plotted with fitted decay profiles (red) for the 11+ 4 charge state of ubiquitin at selected pressures. The decay constants (c) of each fitted decay

5 function illustrate the relationship between the pressure in the Orbitrap analyzer and the transient 6 signal decay rate. 


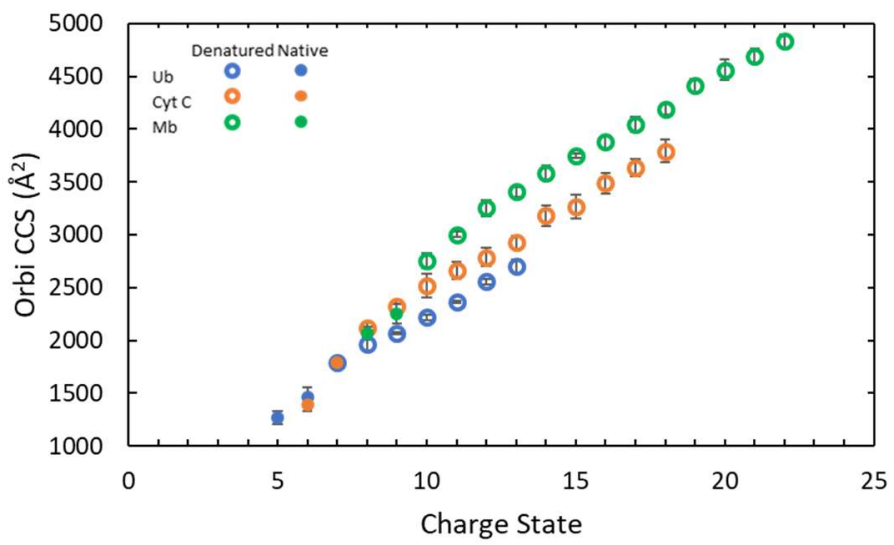

3

$4 \quad$ Figure 3: CCS of protein ions generated from denaturing (hollow circles) or non-denaturing

5 (solid circles) solvent conditions plotted as a function of charge state for three proteins:

6 ubiquitin $(\mathrm{Ub})$, cytochrome $\mathrm{C}(\mathrm{Cyt} \mathrm{C})$, and myoglobin $(\mathrm{Mb})$. Each point represents the average

7 of 5 replicate measurements, and error bars represent \pm 1 standard deviation. 


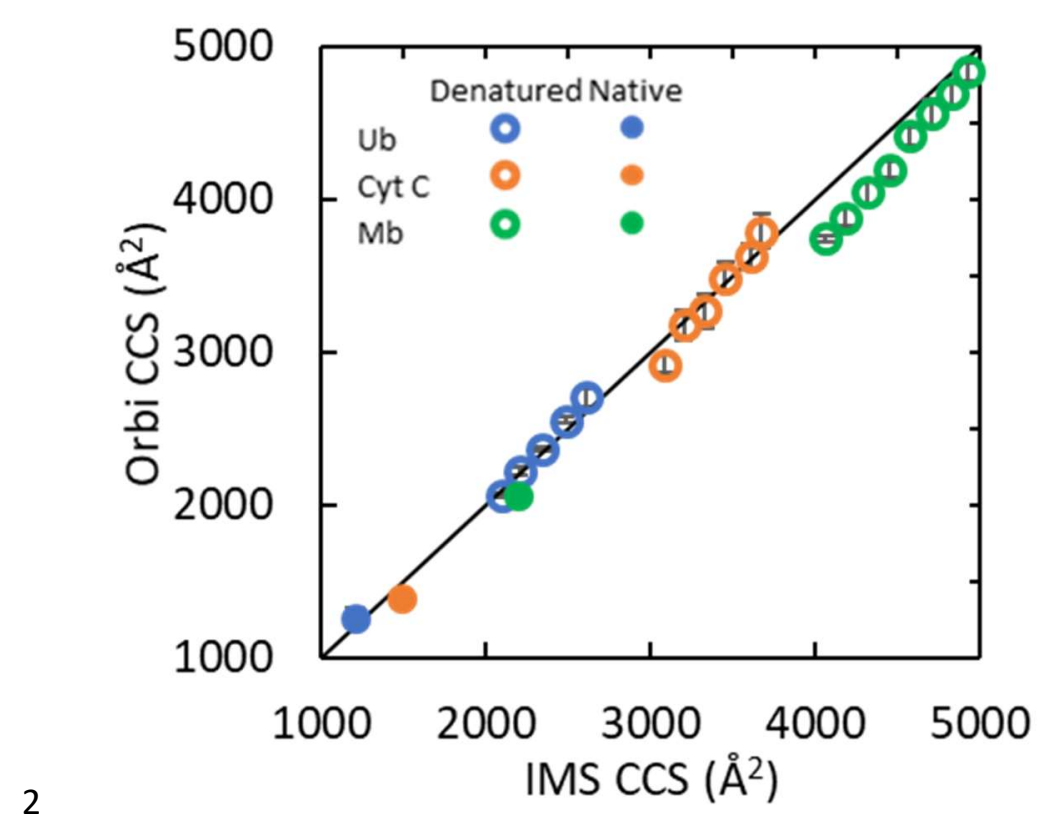

3 Figure 4: Comparison of CCS measured in the Orbitrap (vertical axis) to CCS measured by drift 4 tube IMS (horizontal axis). IMS values from references ${ }^{25,49,56}$ (see Table 1). Each point 5 represents the average of 5 replicate measurements, and error bars represent \pm 1 standard 6 deviation. 
1 Table 1: CCS values measured in this study (Orbi CCS) chosen for comparison with reference 2 CCS values from IMS studies ( ${ }^{\mathrm{a}}$ from,${ }^{56 \mathrm{~b}}$ from, ${ }^{25}$ and ${ }^{\mathrm{c}}$ from $\left.{ }^{49}\right)$. Charge states of ions generated 3 from native-like solutions are indicated by *.

4

\begin{tabular}{lcccccc}
\hline \multirow{1}{*}{ Protein } & $\begin{array}{c}\text { Charge } \\
\text { state }\end{array}$ & $\begin{array}{c}\text { Average } \\
\mathrm{m} / \mathrm{z}\end{array}$ & $\begin{array}{c}\text { IMS } \\
\text { CCS } \\
\left(\AA^{2}\right)\end{array}$ & $\begin{array}{c}\text { Orbi } \\
\text { CCS } \\
\left(\AA^{2}\right)\end{array}$ & $\begin{array}{c}\text { Orbi } \\
\text { CCS } \\
\text { Standard } \\
\text { Deviation }\end{array}$ & $\begin{array}{c}\text { \% } \\
\text { Difference }\end{array}$ \\
\hline Ubiquitin & $5^{*}$ & 1714 & $1204^{\mathrm{a} \dagger}$ & 1271 & 68 & 5.6 \\
& 9 & 953 & $2090^{\mathrm{b}}$ & 2090 & - & - \\
& 10 & 858 & $2200^{\mathrm{b}}$ & 2245 & 30 & 2.0 \\
& 11 & 780 & $2340^{\mathrm{b}}$ & 2394 & 21 & 2.3 \\
& 12 & 715 & $2480^{\mathrm{b}}$ & 2588 & 27 & 4.3 \\
& 13 & 660 & $2600^{\mathrm{b}}$ & 2738 & 59 & 5.3 \\
\hline Cytochrome c & $6^{*}$ & 2060 & $1490^{\mathrm{b}}$ & 1413 & 61 & -5.1 \\
& 13 & 952 & $3080^{\mathrm{b}}$ & 2960 & 70 & -3.9 \\
& 14 & 884 & $3200^{\mathrm{b}}$ & 3218 & 99 & 0.6 \\
& 15 & 825 & $3330^{\mathrm{b}}$ & 3309 & 116 & -0.6 \\
& 16 & 774 & $3450^{\mathrm{b}}$ & 3529 & 99 & 2.3 \\
& 17 & 728 & $3600^{\mathrm{b}}$ & 3674 & 84 & 2.0 \\
\hline Holomyoglobin & 18 & 688 & $3670^{\mathrm{b}}$ & 3837 & 110 & 4.6 \\
\hline Apomyoglobin & 15 & 2197 & $2187^{\mathrm{c}}$ & 2089 & 67 & -4.5 \\
& 16 & 1131 & $4060^{\mathrm{b}}$ & 3791 & 32 & -6.6 \\
& 1060 & $4180^{\mathrm{b}}$ & 3929 & 77 & -6.0 \\
& 17 & 998 & $4310^{\mathrm{b}}$ & 4096 & 82 & -5.0 \\
& 18 & 925 & $4440^{\mathrm{b}}$ & 4241 & 63 & -4.5 \\
& 19 & 893 & $4570^{\mathrm{b}}$ & 4520 & 154 & -1.1 \\
& 20 & 849 & $4700^{\mathrm{b}}$ & 4614 & 111 & -1.8 \\
& 21 & 808 & $4820^{\mathrm{c}}$ & 4749 & 71 & -1.5 \\
& 22 & 772 & $4920^{\mathrm{c}}$ & 4887 & 74 & -0.7 \\
& & & & & &
\end{tabular}

